\title{
Upper gastro-intestinal endoscopy and radiology in the elderly
}

\author{
T. V. Stanley \\ M.B., Ch.B. (Ed.) M.R.C.P. (U.K.)
}

\author{
J. B. COCKING \\ M.B. (Cantab), M.R.C.P.
}

\author{
Isle of Thanet District Hospital
}

\begin{abstract}
Summary
The results of oesophago-gastro-duodenoscopy (OGD) and radiology in one hundred consecutive elderly patients were reviewed to assess the efficacy, safety and acceptability of endoscopy $\ln$ this age group. False positive radiological features occurred in two of nine patients with normal endoscopic examinations. The remaining ninety-one patients had 138 lesions, sixty-four of which were diagnosed by both techniques. Endoscopy missed fourteen lesions partly because of unsuccessful intubation (one patient) or incomplete examinations due to obstructive lesions (eight patients). Radiology misdiagnosed or did not demonstrate sixty-one lesions. OGD proved to be safe and relatively well tolerated; it was invaluable at confirming or refuting the diagnosis of malignancy, at detecting multiple pathology and identifying bleeding lesions, but it gave inferior results with hiatus herniae. Thus it can usefully be undertaken in the elderly as a complementary examination to radiology.
\end{abstract}

\section{Introduction}

The results of oesophago-gastro-duodenoscopy (OGD) and radiology in one hundred elderly patients were reviewed. The study was undertaken by the authors to confirm their clinical impressions that OGD is as valuable in the assessment of upper gastro-intestinal lesions in the elderly as it is in younger patients.

\section{Patients and methods}

Criteria for inclusion in the study were that the endoscopies should have been carried out consecutively and should have been initial examinations, thereby excluding endoscopic retrograde cholangiopancreatography and follow-up examinations; patients should be aged seventy years or older and should have undergone single contrast barium meals within two weeks of endoscopy.

Relative contra-indications to endoscopy were: (1) generalized cachexia; (2) severe nervous disposition; (3) severe skeletal deformity; (4) acute pulmonary infection, heart failure or myocardial infarction; (5) uncontrolled cardiac arrhythmias; (6) chronic pulmonary disease with dyspnoea, and/or excess coughing.

Details of age and sex appear in Table 1; mean age of the group was $75 \pm 0.5$ s.e. mean years. There were fifty-three in-patients and forty-seven outpatients; twenty-eight patients were examined urgently because of acute gastro-intestinal bleeding.

Having given written consent to undergo endoscopy, patients were given a premedication of atropine sulphate $0.6 \mathrm{mg}$ intramuscularly, and asked to suck amethocaine lozenges, 30 min before intubation. They were examined in the left lateral position with an Olympus GIF D2 endoscope, under as light a level of sedation with intravenous diazepam as they would tolerate - the usual dose being $7.5 \mathrm{mg}$; occasionally intravenous hyoscine butylbromide was also used.

TABle 1. Patient data

\begin{tabular}{|c|c|c|c|c|}
\hline & \multicolumn{2}{|c|}{ Male } & \multicolumn{2}{|c|}{ Female } \\
\hline & Numbers & $\begin{array}{c}\text { Mean age } \\
( \pm \text { s.e. } \\
\text { mean })\end{array}$ & Numbers & $\begin{array}{c}\text { Mean age } \\
( \pm \text { s.e. } \\
\text { mean })\end{array}$ \\
\hline Age $70-79$ years & 30 & $73(0 \cdot 4)$ & 53 & $74(0.4)$ \\
\hline Age $80+$ years & 6 & $84(1 \cdot 7)$ & 11 & $84(0.5)$ \\
\hline
\end{tabular}

The final diagnosis given to each patient was derived from the findings at radiology and endoscopy. Lesions diagnosed by radiology in patients whose endoscopic examination was normal were regarded as false positive findings provided that biopsies from the appropriate site showed normal histology. Lesions not demonstrated by barium meal which were seen at endoscopy and confirmed by biopsy were regarded as false negative results or 'missed by radiology'. Other lesions were classified as 'misdiagnosed by radiology' if the endoscopic and histological features differed from those diagnosed from the radiographs. Certain lesions were considered to have been 'missed by endoscopy' if they were indisputably present on the X-ray films, but were not seen during the endoscopic examination.

Results

A comparison of radiology and endoscopy appears 
in Table 2. Nine patients had normal examinations at endoscopy; radiology was in agreement in seven, but produced false positive diagnosis in two, one being misdiagnosed as an oesophageal cancer, the other as a benign gastric ulcer. The remaining ninety-one patients were considered to have 138 lesions, sixty-four of which were detected by both techniques. Thirty-seven lesions were missed by radiology and in this group were seven patients with false negative barium meals, four of whom had gastritis and duodenitis and three of whom had multiple gastric erosions and a hiatus hernia. Twenty-four lesions were demonstrated by radiology, but were incorrectly diagnosed. The other fourteen lesions were missed by endoscopy.

Radiological appearances could be misleading in both benign and malignant conditions. Areas of oesophagitis, gastritis and duodenitis tended to be inadequately demonstrated, resulting in these lesions being missed or incorrectly diagnosed by radiology in seven of nine patients with oesophagitis, nineteen of twenty patients with gastritis and four of six patients with duodenitis. Lesions of the pylorus and pyloric canal also gave rise to features which were difficult to interpret; thus three of seven patients with histologically proved gastritis and superficial ulceration of this area had barium meal findings which were consistent with malignancy. Similar difficulties arose with benign peptic ulcers. There were twenty-three benign gastric ulcers in the series fourteen were diagnosed by radiology, three were् missed, and six others were misdiagnosed; alsaseven of nine chronic duodenal ulcers were not demonstrated by radiology.

Malignant conditions of oesophagus and stomacte proved to be equally difficult to diagnose accurately. by barium studies. There were nine patients with histologically proved oesophageal carcinomata; six were diagnosed by both radiology and endoscopy: whereas one was missed and two were misdiagnosedby radiology. Radiology also made false positive diagnoses of oesophageal cancer in two othe $\vec{\omega}$ patients; one had no lesion seen at endoscopy, and? the other had a benign oesophageal strictureso Gastric carcinomata were present in ten patients six were diagnosed correctly by both diagnostic techniques, whereas four were misdiagnosed byos radiology. Eight other patients had barium meas appearances which were compatible with gastric cancer but malignancy was not confirmed by endos-copy or histology; one patient had gastritis, one haf benign gastric polyps, another had a leiomyoma, two had pyloric canal inflammation and scarring, and three had benign gastric ulcers.

One patient with a gastric carcinoma was mis-e diagnosed initially by both diagnostic methods. Thisol was an unusual lesion situated high on the lesseri curve which looked like an area of gastritis and

TABLE 2. Radiology and endoscopy findings in 100 elderly patients

\begin{tabular}{|c|c|c|c|c|c|c|c|}
\hline & \multicolumn{3}{|c|}{ Diagnosis: } & & & & \\
\hline & \multirow{2}{*}{$\begin{array}{c}\text { (a) } \\
\text { made by } \\
\text { radiology } \\
+ \\
\text { endoscopy }\end{array}$} & \multirow{2}{*}{$\begin{array}{l}\text { (b) } \\
\text { missed by } \\
\text { radiology } \\
\text { but made at } \\
\text { endoscopy }\end{array}$} & \multirow{2}{*}{$\begin{array}{c}\text { (c) } \\
\text { misdiagnosed } \\
\text { by } \\
\text { radiology } \\
\text { but made at } \\
\text { endoscopy }\end{array}$} & \multicolumn{3}{|c|}{ Lesions missed by endoscopy: } & \\
\hline & & & & $\begin{array}{c}\text { (a) } \\
\text { during full } \\
\text { examination }\end{array}$ & $\begin{array}{l}\text { (b) } \\
\text { because of } \\
\text { proximal } \\
\text { obstruction }\end{array}$ & $\begin{array}{c}\text { (c) } \\
\text { because of } \\
\text { unsuccessful } \\
\text { intubation }\end{array}$ & Totals \\
\hline Normal examinations & 7 & 0 & $2 \dagger$ & 0 & 0 & 0 & 9 \\
\hline Oesophagitis & 2 & 7 & 0 & 0 & 0 & 0 & 9 \\
\hline Benign oesophageal stricture & 13 & 0 & 2 & 0 & 0 & 0 & 15 \\
\hline Hiatus hernia & 11 & 1 & 0 & 3 & 8 & 1 & 24 \\
\hline Oesophageal diverticulum & 2 & 0 & 0 & 0 & 0 & 0 & 2 \\
\hline Carcinoma of oesophagus & 6 & 1 & 2 & 0 & 0 & 0 & 9 \\
\hline Benign gastric ulcer & 14 & 3 & 6 & 0 & 0 & 0 & 23 \\
\hline Gastritis \pm erosions & 1 & 14 & 5 & 0 & 0 & 0 & 20 \\
\hline Gastric polyps & 0 & 0 & 1 & 0 & 0 & 0 & 1 \\
\hline Leiomyoma & 0 & 0 & 1 & 0 & 0 & 0 & 1 \\
\hline Carcinoma of stomach & 6 & 0 & $4^{*}$ & $1^{*}$ & 0 & 0 & 10 \\
\hline \multicolumn{8}{|l|}{ Pyloric canal inflammation and } \\
\hline deformity & 4 & 0 & 3 & 0 & 0 & 0 & 7 \\
\hline Chronic duodenal ulcer & 2 & 7 & 0 & 0 & 0 & 0 & 9 \\
\hline Duodenitis \pm scarring & 2 & 4 & 0 & 0 & 0 & 0 & 6 \\
\hline Duodenal polyps & 1 & 0 & 0 & 0 & 0 & 0 & 1 \\
\hline Duodenal diverticula & 1 & 0 & 0 & 0 & 1 & 0 & 1 \\
\hline \multicolumn{8}{|l|}{$\begin{array}{l}\text { Totals (excluding normal } \\
\text { examinations) }\end{array}$} \\
\hline
\end{tabular}


not biopsied. A follow-up examination was performed which is not included in this series, and the patient underwent surgery.

Endoscopy gave indifferent results in the diagnosis of hiatus herniae. There were twenty-four patients who could be made to herniate, and in twelve endoscopy failed to show the lesion; in three patients the lesion was not seen at a full examination of the upper gut, in one intubation failed and in eight there were oesophageal strictures which could not be negotiated by the endoscope. The only other lesion demonstrated by radiology which was not seen at endoscopy was a duodenal diverticulum in a patient with pyloric canal narrowing, through which the endoscope could not be passed.

The accuracy of radiology in patients with multiple pathology is illustrated in Table 3. The patients have been subdivided into three groups; one in which the radiological diagnoses were correct, one in which one or more lesions were correctly diagnosed but other lesions were missed or misdiagnosed, and a third group in whom all lesions were missed or incorrectly diagnosed. There were fifty-three patients in whom the radiological diagnosis was correct, fourteen patients were in the partially correct group, and in thirty-three the radiological diagnoses were incorrect. Radiology was most inaccurate in patients with multiple pathology; thirty-four patients had two or more lesions, of whom only eleven were correctly diagnosed; in twenty-three radiology produced either partially or completely incorrect information.

Twenty-eight patients in this series had suffered with acute gastro-intestinal bleeding. Radiology was approximately $50 \%$ accurate at diagnosing single bleeding lesions, but was again less accurate when there was multiple pathology; thirteen of the patients who bled were in the latter category and in ten of them the radiological diagnoses were inaccurate.

Two patients had undergone previous partial gastrectomies. These features were visualized by both endoscopy and radiology and no stomal ulceration was seen.

\section{Difficulties and complications of endoscopy}

Seven patients complained of pain at the site of the intravenous diazepam injection, and six of sore throats following the examination. One patient disliked the examination to the extent of refusing to have it repeated should this have been necessary. Although intubation can be slow in the elderly, in three patients it was exceptionally difficult and in another it was unsuccessful. The trachea was entered once during intubation, surprisingly without producing any respiratory embarrassment. Temporary respiratory depression occurred for a few minutes in another patient following the diazepam injection. One patient who suffered with mild bronchial asthma developed increasing wheeze throughout the procedure which responded to intravenous hydrocortisone. Two patients vomited but no inhalation occurred. There is no evidence that inhalation into the lungs occurred in anyone in this series; sedation was deliberately kept very light to prevent this. Finally the gums of one edentulous patient punctured the protective sheath of the endoscope early in this study. Following this incident, a policy of using mouthpieces in all patients was adopted.

\section{Discussion}

This study has shown that fibreoptic endoscopy is of considerable value in the detection of upper gastrointestinal tract pathology in the elderly. It proved to be a safe and relatively well tolerated procedure in this age group provided that medically unsuitable patients were rejected at the outset. These findings confirm the observations of Belsito and Dickinson (1972).

There were several clinical situations in which it improved the accuracy of the radiological diagnosis. Thus false positive appearances were present on the barium meals of two out of nine patients with normal endoscopic examinations, and conversely seven patients with dyspepsia and negative barium meals were found to have organic disease at endoscopy. Radiology gave poor visualization of superficial mucosal lesions of oesophagus, stomach and duodenum, and failed to demonstrate duodenal ulceration in seven of nine patients because of concomitant scarring and deformity of the duodenal cap. However, these observations give less cause for concern than the errors that were made in the diagnosis by

TABLE 3. Radiology and multiple pathology, including those with haemorrhage

\begin{tabular}{|c|c|c|c|c|c|}
\hline & & \multicolumn{4}{|c|}{ X-ray groups } \\
\hline & & Correct & $\begin{array}{l}\text { Partially } \\
\text { correct }\end{array}$ & Incorrect & Totals \\
\hline \multirow[t]{2}{*}{ Patient groups } & $\left\{\begin{array}{l}\text { No lesion } \\
\text { One lesion } \\
\text { Two or more lesions }\end{array}\right.$ & $\begin{array}{l}7 \\
35(7)^{*} \\
11(3)\end{array}$ & $\begin{array}{r}0 \\
1 \\
13(7)\end{array}$ & $\begin{array}{l}2 \\
21(8) \\
10(3)\end{array}$ & $\begin{array}{l}9 \\
57(15) \\
34(13)\end{array}$ \\
\hline & Totals & $53(10)$ & $14(7)$ & $33(11)$ & $100(28)$ \\
\hline
\end{tabular}

*Figures in parenthesis relate to patients with haemorrhage. 
radiology of gastric lesions particularly of the pyloric region, which resulted in some malignant lesions being labelled as benign and some benign ones as malignant.

The radiological appearances of malignant conditions in general gave difficulties in interpretation. Ten patients had false positive radiological features which incorrectly suggested that malignancy was present, and a further nineteen patients had proved oesophageal or gastric cancers, of whom seven were misdiagnosed by radiology. This emphasizes the importance of a complementary examination by endoscopy to visualize lesions directly and obtain biopsies for histological examination. It was for this reason that endoscopy was essential in the management of oesophageal strictures. Radiology correctly diagnosed thirteen of fifteen benign oesophageal strictures, but biopsies were needed in all cases to confirm the diagnosis.

Multiple pathology of the upper gut was another situation in which endoscopy was required for completeness of diagnosis. One third of the patients had two or more lesions, and of these only one third were correctly diagnosed by radiology. Endoscopy was also found to be more effective than radiology at detecting bleeding lesions, especially in the presence of other pathology. Twenty-eight patients had bled and thirteen of them had two or more lesions; of this latter group only three patients were fully diagnosed by radiology. Stevenson, Cox and Roberts (1976), have shown that the accuracy of radiology is improved by the use of double-contrast barium meals but that it is still less accurate than endoscopy, which remains the investigation of choice in the bleeding patient; they found that endoscopy was more accurate than double contrast radiology, "was no more unpleasant than a barium meal examination and it was less tiring for the elderly and immobile'.

In contrast, there were certain patients in whom radiology was of more value than endoscopy. In one patient intubation was unsuccessful and in nine other patients endoscopic examinations were incompletez because of stenoses of oesophagus or pylorus through which the instrument would not pass; eight. hiatus herniae and one duodenal diverticulum were missed by endoscopy because of these obstructions $\stackrel{?}{?}$ Four other lesions were not seen during full endo은 scopic examinations; three were hiatus herniae and $\frac{\bar{c}}{6}$. the fourth was a high lesser curve gastric neoplasm? which was also not detected by radiology. Thea superiority of radiology over endoscopy in the authors' hands at demonstrating hiatus herniae waspartly because no attempt was made to produceherniation during endoscopic examinations, whereas $\vec{\omega}$ this was done routinely by the radiologists. Also, hiatus herniae are probably more easily identified ino profile views where the relationship of the hernia to 3 . the diaphragm can be seen.

Both this retrospective work and the prospective investigation of Stevenson et al. (1976) suggest thato endoscopy by direct visualization and biopsy of lesions gives a more accurate assessment than radiology. The authors have shown that it is the investigation of choice in patients with upper gastric intestinal bleeding and is of especial value in con-o firming or refuting the presence of malignant disease. However, as the information yielded by endoscogy $\vec{\theta}$ to a certain extent complements the barium mealos findings, both procedures should be undertaken हfं the elderly to establish a comprehensive and accurate diagnosis.

\section{Acknowledgment}

The manuscript was typed by Mrs R. Bowles.

\section{References}

Belsito, A.A. \& Dickinson, P. (1972) Fiberoptic esophagogastroscopy. A crucial diagnostic test in the elderly. Geriatrics, 27, 90.

Stevenson, G.W., Cox, R.R. \& Roberts, C.J.C. (1976) Prospective comparison of double-contrast barium mealexamination and fibreoptic endoscopy in acute upper $\overline{3}$ gastrointestinal haemorrhage. British Medical Journal, 2, 723. 